\title{
Soil mediates the interaction of coexisting entomopathogenic nematodes with an insect host
}

\author{
Daniel S. Gruner *, Karthik Ram, Donald R. Strong \\ Bodega Marine Lab and Section of Evolution \& Ecology, University of California-Davis, P.O. Box 247, Bodega Bay, CA 94923-0247, USA
}

Received 26 April 2006; accepted 15 August 2006

Available online 26 September 2006

\begin{abstract}
We tested for soil substrate effects on the movement and infectivity of naturally co-occurring entomopathogenic nematodes Steinernema feltiae and Heterorhabditis marelatus, alone and in combination. We manipulated the presence and bulk density of soil and added Galleria mellonella baits within capped and perforated $15 \mathrm{~mL}$ centrifuge tubes. Sampling tubes were then deployed in situ into field and laboratory settings as experimental traps for infective juveniles. In comparisons with standard soil collections from Lupinus arboreus rhizospheres, sampling tubes were equally sensitive to the presence of $H$. marelatus and more sensitive to $S$. feltiae. In laboratory microcosms, both EPN species infected Galleria at high frequencies in tubes lacking soil and in the absence of heterospecifics. Infection frequency of $S$. feltiae was unaffected by the presence of $H$. marelatus, but it declined with higher soil bulk density inside tubes. In contrast, detectable infection frequency by $H$. marelatus was reduced only marginally by the presence of soil but severely by the presence of $S$. feltiae. Thus, the presence of soil in tubes reversed the identity of dominant species infecting Galleria in tubes, an effect magnified when soils were compacted. Moreover, $S$. feltiae rarely moved into tubes lacking Galleria baits, whereas H. marelatus colonized unbaited tubes 4- to 5-fold more frequently than S. feltiae. In situ, sampling tubes acted as filters to reduce interference and contamination by fungal pathogens common in field soils. The method allows precision sampling with minimal soil disturbance while protecting bait insects from scavengers. Manipulation of tube design may allow selective sampling of EPN species, depending on the abiotic characteristics of soils, and the biology, behavior, and interspecific interactions of coexisting species.
\end{abstract}

(C) 2006 Elsevier Inc. All rights reserved.

Keywords: Bedding and Akhurst bait method; Bodega head; California; Entomopathogenic nematodes; Foraging behavior; Galleria mellonella; Heterorhabditis marelatus; Interspecific interactions; Soil bulk density; Soil moisture; Sampling technique; Steinernema feltiae

\section{Introduction}

Foraging dauer stage entomopathogenic nematodes (EPNs) often move toward carbon dioxide and other volatile chemical compounds released by host insects, plant roots, and organic substances in soils (Gaugler et al., 1980; Lewis et al., 1992, 1993; O'Halloran and Burnell, 2003; Rasmann et al., 2005). Dauer stage EPNs (also referred to as infective juveniles, 'IJs') can be attracted to excretions, surface washes and feces of host or bait insects, such as larvae of the greater wax moth Galleria mellonella (Schmidt and All, 1978, 1979; Hui and Webster, 2000). Attractiveness of hosts varies as a

\footnotetext{
* Corresponding author. Fax: +1 7078752009.

E-mail address: dsgruner@ucdavis.edu (D.S. Gruner).
}

function of host species or strain, physiological condition, and prior parasitism (Grewal et al., 1997; Gouge et al., 1999; Hui and Webster, 2000). IJ foraging also may be modulated by abiotic conditions, such as soil temperature or moisture content (Kung et al., 1991; Brown and Gaugler, 1997), or soil physical properties, such as soil texture, pore size and bulk density (Georgis and Poinar, 1983a,b; Portillo-Aguilar et al., 1999; Koppenhöfer and Fuzy, 2006). Although many of these factors have been explored in laboratory experiments (e.g., Barbercheck and Kaya, 1991), we lack a comprehensive understanding of how these factors mediate the emergent behavior and virulence of entomopathogenic nematodes under natural field conditions.

Before Bedding and Akhurst (1975), EPNs typically were isolated from soils by searching for parasitized insect 
cadavers in the field - where they are highly patchy and ephemeral - or extracted directly from soils, isolated, and counted (Hass et al., 1999). However, extraction or flotation methods are labor intensive and can be problematic because IJs represent a small fraction of nematodes found in typical environments and can be difficult to distinguish from these similar organisms in samples (e.g., Neher et al., 2004). Bedding and Akhurst (1975) proposed the use of bait insects (e.g., the greater wax moth, Galleria mellonella [L.]) to sample IJs from collections of soil. Their contribution represented a major advance by harnessing the chemotactic behavior of IJs to quickly and easily concentrate nematodes from the background soil matrix.

The infection rate in bait insects is a reliable and sensitive indicator of the magnitude of risk. Direct counts are seldom used because IJs are temporally and spatially variable in local incidence, viability, and infectivity to hosts, and their low natural abundance relative to other nematode functional groups hinders their successful extraction (Woodring and Kaya, 1998). Typical to studies of microparasites and diseases (Anderson and May, 1981), this approach also lends itself to modeling EPN-host dynamics in an epidemiological context. Several studies have shown that EPN infection rates are correlated with IJ counts under controlled conditions (Fan and Hominick, 1991; Koppenhöfer et al., 1998; Hass et al., 1999; Kehres et al., 2001; Nielsen et al., 2004). Baiting techniques provide an expedient, accurate, and conceptually sound snapshot of the landscape of EPN infection risk to hosts in the field.

Building upon the scheme proposed originally by Bedding and Akhurst (1975), we present a sampling design that can be used to detect EPNs in situ in field and laboratory settings. Although Bedding and Akhurst (1975) proposed in situ use of their method, they did not enclose bait insects, which then become difficult to relocate or lost to predators, scavengers and pathogens. Subsequent workers introduced modified in situ sampling techniques using screen cages (McCoy et al., 2000) or perforated microcentrifuge tubes (Kehres et al., 2001) as protected sampling devices. We offer an incremental improvement of these methods that allows experimentation of soil and host factors in situ, with modified sampling tubes that are both easily mass produced and readily relocated in heterogeneous field settings.

We evaluated the sampling tubes using both laboratory and field experiments at the University of California laboratory on the Bodega Marine Reserve, a 147-ha parcel comprised of coastal dunes and mixed annual grass and shrublands $\left(38^{\circ} 19^{\prime} \mathrm{N} 123^{\circ} 04^{\prime} \mathrm{W}\right.$; Barbour et al., 1973). Two species of entomopathogenic nematodes have been isolated from the soils on the Bodega Marine Reserve, one each from the families Steinernematidae and Heterorhabditidae. Heterorhabditis marelatus Liu \& Berry is a species native to the Pacific Coast of California and Oregon (U.S.A.), and Steinernema feltiae (Filipjev) is a cosmopolitan species often found in grasslands (Stock et al., 1999).

We show that in situ sampling tubes were equivalent $(H$. marelatus) or more sensitive (S. feltiae) than soil collections when sampling natural populations of IJs. Moreover, mortality of bait insects by entomophagous fungi and other unexplained causes was higher in baited soil collections than in tubes. In situ sampling tubes thus act as filters to reduce interference and contamination by pathogens that are ubiquitous in field collected soils but which cannot actively pursue hosts. Moreover, by varying the presence and bulk density of soils within the tubes, we show that soil properties mediate interspecific interactions and reverse the relative infection frequencies of $H$. marelatus and $S$. feltiae in Galleria. These design modifications may allow selective capture of different nematode species which vary in their sensitivity to host- and non-host cues, their ability to move through soil media, and their infectivity to various host taxa.

\section{Materials and methods}

\subsection{In situ baiting technique}

In order to evaluate the efficacy of in situ baiting, and to compare it with the typical soil collections method (Bedding and Akhurst, 1975), we modified $15 \mathrm{~mL}$ graduated centrifuge tubes $\left(\mathrm{BD}^{\circledR}\right.$ Falcon $^{\circledR}$ BlueMax ${ }^{\circledR} \mathrm{Jr}$. polystyrene, item\# 352099; $12 \mathrm{~cm}$ length, $2 \mathrm{~cm}$ diameter) for use in laboratory and field experiments. To allow gas exchange and entry of foraging IJs, we used a Dremel ${ }^{\circledR}$ tool (Robert Bosch Tool Corporation, Mt. Prospect, IL) to drill a single small hole (1-2 mm diam.) through the tube bottoms and six holes around the lip of the tapered base $1 \mathrm{~cm}$ above the bottoms (Fig. 1). Bait insects (Galleria mellonella larvae) were stocked inside tubes, which were then capped and deployed into soils as a probe for the presence of EPN infective juveniles. For all treatments, we used two Galleria larvae to reduce the chance of losing replicates to occa-
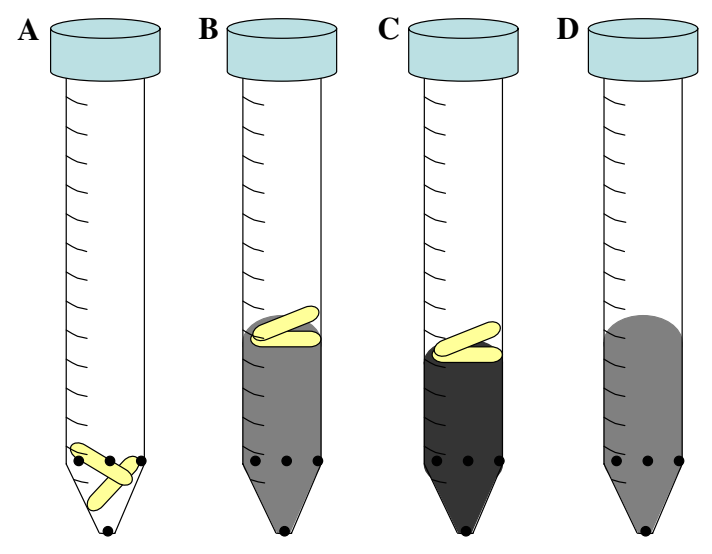

Fig. 1. Cartoon of $15 \mathrm{~mL}$ graduated centrifuge tubes modified for in situ field sampling of entomopathogenic nematodes. To allow gas exchange and ingress of foraging nematodes, we drilled six 1-2 mm holes around the lip of the tapered base and one hole through the bottom of each tube. The four tube treatment levels were: (A) baited with 2 Galleria larvae, no soil; (B) baited, saturated soil; (C) baited, packed and saturated soil; (D) no bait, saturated soil. Soil was isolated from field site, sifted, pasteurized, then saturated with distilled water; all baits consisted of two Galleria larvae. Treatment level (D) was baited after removal from soil medium. 
sional unexplained Galleria mortality. Individual tubes were treated as the unit of replication with all Galleria responses measured as binary variables. For brevity, henceforth we use the term "tubes" to indicate this in situ sampling technique.

The following treatments were deployed in capped tubes for both laboratory and field experiments (Fig. 1):

(A) Baited, no soil. No soil was added to tubes, but tubes were baited with 2 Galleria larvae;

(B) Baited, with saturated soil. $6 \mathrm{~cm}^{3}$ of dry, pasteurized soil was added to tubes and wetted by wicking to saturation in baths of distilled water. Two Galleria were added to each tube after draining excess moisture for one hour;

(C) Baited, packed and saturated soil. As in (B) above, but the bulk density of soil was increased by packing the wetted soil in tubes with a $1 \mathrm{~cm}$ diameter probe. After packing the tubes, tubes were soaked again in water baths to restore to saturation;

(D) No bait, saturated soil. Identical to wetted treatment (B), but without Galleria hosts. This treatment tested whether entomopathogenic nematodes recruit to saturated soils in the absence of host cues.

We used soil from lower Mussel Point, an area of the reserve where both nematode species have been collected (Strong et al., 1996; Gruner et al. unpublished data), to fill tubes and for all lab experiments. This soil, classified as a loamy sand (Miller, 1972; 81:7:12 sand:silt:clay), was collected from beneath the grass root layer and sifted through a mesh sieve to remove vegetative matter, stones, and large soil clumps $(0.5 \times 0.5 \mathrm{~cm}$ mesh). Soil was then pasteurized at $60^{\circ} \mathrm{C}$ and dried to constant mass to sterilize soil without changing its physical properties. In both field and laboratory evaluations, soil moisture from randomly selected tubes was measured using standard gravimetric techniques at the outset and conclusion of experiments, both inside tubes and in the background soil medium surrounding tubes. To sample soil from inside tubes, we used a spatula to extract approximately $1 \mathrm{~g}$ of soil. We measured bulk density of soils by reading volume graduations $\left(\mathrm{cm}^{3}\right)$ on the tubes and obtaining wet and dry mass of total soil volumes.

\subsection{Field tests and comparison with soil collection technique}

Field investigations were run in December 2005 in an area on Mussel Point where both nematode species were present, although $S$. feltiae was the most locally abundant species. We used 121 experimental rhizospheres, each planted with a single yellow bush lupine (Lupinus arboreus Sims) in March-April of 2004 as part of a larger experiment. To provide a direct comparison of all tube treatment levels to soil collection methods, we first collected $100-\mathrm{cm}^{3}$ soil samples from each rhizosphere into 8-oz lidded deli cups (Solo Products ${ }^{\circledR}$ item\# DM8). Soil was taken from the first $5 \mathrm{~cm}$ of topsoil beneath the litter or grass layer. We then inserted four modified centrifuge tubes, corresponding to the treatment levels above, in random positions around each lupine. Tubes were planted such that their blue caps were plainly visible at the soil surface and their entry holes were exposed $7-10 \mathrm{~cm}$ below the surface. Soil collections were incubated at ambient temperature for 10 days with two Galleria larvae. Tubes were collected after seven days in the field, and incubated out of soil at ambient temperature for an additional three days. Two Galleria were then added to all tubes in the bait control treatment (Fig. 1D; no bait, saturated soil), and these larvae were incubated for 10 days before analysis. Average ambient air temperatures (Bodega Marine Laboratory, unpublished data) and soil temperatures at $10 \mathrm{~cm}$ depth (J. Bastow, unpublished data) during this period were similar $\left(10-14^{\circ} \mathrm{C}\right)$, although the range in air temperatures was greater.

We recorded five possible outcomes for Galleria larva in the tubes and in the soil collection cups: positive for $H$. marelatus, $S$. feltiae, or fungi; dead without attributable cause; or alive. Heterorhabditis marelatus was easily diagnosed by the red-gold color of infection by the symbiotic bacterium Photorhabdus, and Steinernema feltiae was diagnosed by color (grey to copper-brown) followed by dissection. These two species are the only EPNs ever isolated from Bodega marine reserve (Stock et al., 1996; Strong et al., 1996; Stock, 1997; Stock et al., 1999); S.P. Stock, personal communication). Fungal infection of Galleria was diagnosed by observing mycelia or the characteristic hardening caused by entomophagous fungi (EPF: Beauveria bassiana and spp.). Remaining Galleria larvae were either alive or dead by a failed EPN infection or some other unknown cause.

\subsection{Laboratory microcosms}

In laboratory evaluations, we tested for the effects of tube sampling design on each species alone and in the presence of the second species. We used 32-oz plastic deli containers (Solo Products ${ }^{\circledR}$, item\# DM32; $15 \mathrm{~cm}$ depth, $9 \mathrm{~cm}$ diameter at base), each filled with $600-\mathrm{cm}^{3}$ of moist, pasteurized soil (collected and treated as above). We moistened soil to approximately $15 \%$ water content and homogenized large batches to reduce variation among replicates. Water potential in these soils increases rapidly to an inflection point at approximately $10 \mathrm{kPa}$ and $10 \%$ water content, but further changes to water potential are negligible between $10-50 \%$ (Jaffee and Strong, 2005). Four tightly capped centrifuge tubes, one from each of the above treatments, were planted into each deli container at the same depth used in field mesocosms. Soil was then inoculated with infective juveniles in 1-mL pipette aliquots in four separate treatment levels:

100:0 - Added $100 \mathrm{H}$. marelatus IJs to soil; 0:100 - Added 100 S. feltiae IJs to soil; 50:50 - Added $50 \mathrm{H}$. marelatus IJs + 50 S. feltiae IJs, to control for overall density; 
100:100 - Added $100 \mathrm{H}$. marelatus IJs +100 S. feltiae IJs, to control for density of each EPN species.

Each EPN treatment was delivered in 1-mL pipette aliquots with a total of 50 replicates per treatment. Infective juveniles were isolated from wild populations and cultured through two or more generations in Galleria; all IJs were less than 3 weeks old when used in experiments. To estimate the actual inoculation densities, numbers of IJs were counted from random aliquots. Two-sample $t$-tests showed that inoculation densities were similar for each species within treatment levels (Table 1). Laboratory microcosms were in place for eight days following the addition of IJs, then incubated for an additional four days out of soil before analysis for infection. At this time, two Galleria were added to the bait control treatment (Fig. 1D; no bait, saturated soil) and incubated at ambient temperature for ten days.

\subsection{Statistical analysis}

Background soil moisture in field and lab experiments was compared from initial and final (seven days) time periods using two-tailed, two-sample $t$-tests, with the unequal variance assumption when necessary. We used one-way ANOVA to test for initial differences in soil moisture and soil bulk density within tubes across treatments. To test final soil moistures within tubes, we used two-way ANOVA with interaction, treating sampling period (initial and final) and tube treatment as fixed factors. Repeated measures analyses were not applicable because different, randomly selected tubes were sampled at initial and final time periods. Because residuals from all models were normally distributed, we did not transform response variables.

Galleria symptomology from the experiments was analyzed in two steps. Five alternative outcomes (H. marelatus, $S$. feltiae, fungi, dead, alive) from the field experiment and the one-species lab treatments were compared among treatments using simple chi-square contingency table analyses. For the lab experiment, there were four levels of the tube treatment (levels A-D in Section 2.1 above), analyzed in $2 \times 4$ tables for each outcome. In the field experiment, bait-

\section{Table 1}

Estimated inoculation densities (+ 1 SD) for lab microcosm experiments and results from 2-sample, 2-sided Welch $t$-tests

\begin{tabular}{clllll}
\hline EPN ratio & H. marelatus $(\mathrm{SD})$ & S. feltiae $(\mathrm{SD})$ & $t$ & $\mathrm{df}$ & $P$ \\
\hline $100: 0$ & $95.47(10.76)$ & - & 0.098 & 17.67 & 0.923 \\
$0: 100$ & - & $94.67(29.60)$ & & & \\
$50: 50$ & $44.70(8.92)$ & $47.90(9.84)$ & -0.762 & 17.63 & 0.456 \\
$100: 100$ & $105.9(8.53)$ & $100.3(16.49)$ & 0.3568 & 13.50 & 0.357 \\
\hline
\end{tabular}

EPN ratios show the intended IJ densities for $H$. marelatus and $S$. feltiae, respectively. $T$-tests evaluate the null hypothesis that densities of the two species within the treatment do not differ; the first test compares estimated inoculation densities among the one-species treatments. ing results from soil collections were added as a fifth treatment level $(2 \times 5)$, and each of the five categorical outcomes were analyzed in a separate contingency table. For lab experiments, we analyzed results from $H$. marelatus (100:0) and $S$. feltiae $(0: 100)$ as separate sets.

The second stage of the analysis focused on the effect of the conspecific EPN species on the infection frequency of the first species across treatments. Thus, we compared results from the one-species microcosms to the low and high density 2 -species microcosms using generalized linear modeling, with the binomial response transformed with the logit link-function. Tube treatment (A-D), heterospecific inoculation density $(0,50,100)$, and their interaction were treated as fixed factors and evaluated with $F$-tests using type III sums-of-squares. We also included a block term as a fixed effect in models to account for any correlated variation within individual microcosm units.

All analyses were run in the $\mathrm{R}$ package (http://www. r-project.org/).

\section{Results}

\subsection{Soil characteristics}

Water content in background soils did not change over the duration of the lab and field experiments (Fig. 2; field mean $\pm 1 \quad$ S.D $=9.11 \% \pm 2.86, \quad t$-value $=0.95, \quad P=0.356$, $\mathrm{df}=18$; lab mean $=14.04 \pm 1.50, t=0.86, P=0.410, \mathrm{df}=9$ ). Within tubes, compacted soils (Fig. 1C) had higher initial bulk density (compacted mean: $1.35 \mathrm{~g} / \mathrm{cm}^{3} \pm 0.05$, pooled non-compacted mean: $1.21 \mathrm{~g} / \mathrm{cm}^{3} \pm 0.05, t=9.39, P<0.001$, $\mathrm{df}=58$ ). All soils within tubes initially were saturated with moisture, but compacted soils held less moisture (mean loose soil $=27.6 \% \pm 0.9$, compacted $=23.9 \% \pm 1.0$; one-way ANOVA, $\left.F_{2,27}=43.23, P<0.001\right)$. Soils within tubes initially were more moist than background soils, but moisture decreased over the course of both field and lab experiments (time factor, two-way ANOVA, field: $F_{1,54}=351.88$, $P<0.0001$; lab: $\left.F_{1,54}=535.33, P<0.0001\right)$. The overall treatment means did not differ over the course of the experiments, but treatment levels interacted significantly with time in both venues (time $\times$ treatment, field: $F_{2,54}=23.55$, $P<0.001$; lab: $\left.F_{2,54}=15.98, P<0.001\right)$. Tubes converged to background conditions in the lab (Fig. 2B), with modest differences among treatments (mean loose soil = $15.8 \% \pm 3.6, \quad$ compacted soil $=18.5 \% \pm 2.7 ; \quad$ one-way ANOVA, $\left.F_{2,27}=4.09, P=0.03\right)$. Moisture levels in tubes in the field remained higher relative to the background (Fig. 2A) and maintained significant differences in packed and unpacked tube soils (mean loose soil $=15.0 \% \pm 2.8$, compacted soil $=18.9 \% \pm 1.9$; $\quad$ one-way ANOVA, $F_{2,27}=8.33, P=0.002$ ).

\subsection{Field experiment}

In field experiments, H. marelatus was isolated in similar, low proportions across all tube treatments and the soil col- 
A

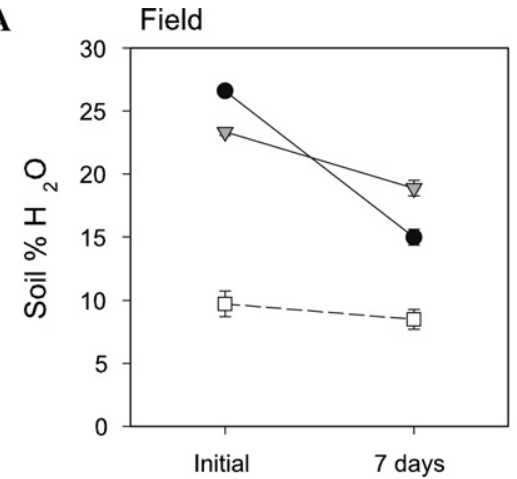

B

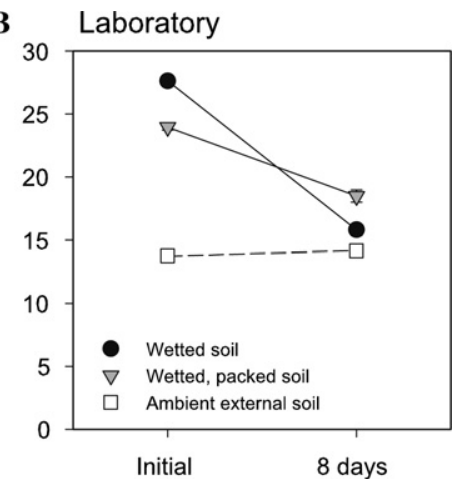

Fig. 2. Percentage gravimetric soil moisture content $( \pm$ S.E. $)$ in tube treatment levels before and after deployment into field and lab sampling units compared to background soil moisture. Tubes without soil and field soil collection treatments are not shown, and both unpacked, wetted soil treatment levels (Fig. 1, B and D) were pooled after finding no difference in initial or final moisture content.

lections $\left(10-15 \%\right.$; Fig. $\left.3 \mathrm{~A} ; \chi^{2}=2.296, \mathrm{df}=4, P=0.681\right)$. Results for $S$. feltiae were more complex (Fig. 3B). We recovered a lower rate of $S$. feltiae infection from soil collections $(36 \%)$ than from baited tube treatments without soil $(60 \%)$ and with wetted loose soil $(55 \%)$, but the largest contributor to the significant differences among treatments was attributable to low recovery in unbaited tubes $(15 \% ; S$. feltiae, $\left.\chi^{2}=61.508, \mathrm{df}=4, P<0.001\right)$. No fungal infections were recorded from 484 tubes outplanted in field rhizospheres, although $18 \%$ of 121 soil collections were positive for fungi (Fig. 3C; $\chi^{2}=91.321, \mathrm{df}=4, P<0.001$ ). Moreover, Galleria were 16-36\% more likely to die of unknown cause and $32-66 \%$ less likely to survive than Galleria from fieldbaited tubes (Fig. 3D, death: $\chi^{2}=50.926, \mathrm{df}=4, P<0.001$; Fig. 3E, survival: $\chi^{2}=120.261$, $\mathrm{df}=4, P<0.001$ ).

\subsection{Laboratory microcosms}

In one-species laboratory microcosms (Fig. 4), both EPN species showed differences in infection frequencies across tube treatments $\left(H\right.$. marelatus, $\chi^{2}=21.852, \mathrm{df}=3$, $P<0.001 ;$ S. feltiae, $\chi^{2}=54.286, \mathrm{df}=3, P<0.001$ ). Both species infected Galleria in virtually all replicates in tubes without soil (H. marelatus: $82 \%$; S. feltiae: $92 \%$ ) and in tubes with loose, saturated soil (H. marelatus: $70 \% ; S$. feltiae: $86 \%)$. H. marelatus also infected a comparable proportion in compacted soils $(66 \%)$, while $S$. feltiae performed less well in compacted soils (46\%). H. marelatus also colonized, and later infected Galleria, in 32\% of the tubes containing no baits, but this occurred in only $8 \%$ of the Steinernema feltiae replicates. Considering the last three categories of outcomes for host larvae, there was only one occurrence of fungal infection, dead Galleria showed little pattern across treatments, and the frequencies of alive Galleria were inversely related to EPN infection frequencies (Fig. 4).

The relative importance of soil properties and heterospecific densities differed for $H$. marelatus and $S$. feltiae responses (Table 2; Fig. 4). H. marelatus did not alter the infection frequency of $S$. feltiae in the high density trials. Infection frequency of $S$. feltiae was reduced in low density trials, relative to single- and two-species high density trials, only in loose soil $(\Delta 40 \%)$ and compacted soil treatments $(\Delta$ $30-36 \%$ ). By contrast, $H$. marelatus infection was strongly, negatively affected by $S$. feltiae in tubes without soil regardless of density $(\Delta 78-80 \%)$, but the negative effect diminished in loose soil $(\Delta 32-44 \%)$ and compacted soil treatments $(\Delta 6-22 \%)$. The highly significant interaction between the tube treatment and $S$. feltiae density on $H$. marelatus (Table 2) showed that compacted soil mitigated this negative effect, and there was a trend towards increasing colonization of unbaited soil in the presence of $S$. feltiae ( $\Delta 8-24 \%$; Fig. 4A). The blocking variable for microcosms was not significant in either model (Table 2).

\section{Discussion}

Soil parameters such as texture, bulk density, $\mathrm{pH}$, organic content and soil water potential can affect IJ behavior, survival, and infectivity to hosts (Portillo-Aguilar et al., 1999; Koppenhöfer and Fuzy, 2006). Manipulation of the presence and bulk density of soil within modified centrifuge tubes designed for in situ sampling revealed new ecological insights on two co-occurring EPN species, Heterorhabditis marelatus and Steinernema feltiae. We expected fewer EPN infections in the tubes with compacted soils, but observed a strong depressive effect only with $S$. feltiae and no effect on $H$. marelatus. High bulk density can impede nematode movement because the total soil porosity or the maximum individual pore spaces can be too small to allow efficient movement (Kung et al., 1990; Portillo-Aguilar et al., 1999; Hunt et al., 2001). The IJs of these two species are similar in average length and width (Poinar, 1990; Stock, 1997), so behavioral differences may play a larger role than intrinsic physical differences. Blackshaw and Senthamizhselvan (1991) showed that the foraging efficiency of $S$. feltiae is sensitive to particle grain size, with maximum activity occurring in sandy soils of intermediate grain size $(700$ $800 \mu \mathrm{m})$. The reduced pore space could hinder the transmission of carbon dioxide or volatile exudates which the nematodes use as host-finding cues. The negative effect of increased bulk density on $S$. feltiae was greater in the presence of $H$. marelatus, suggesting this obstacle mediated 

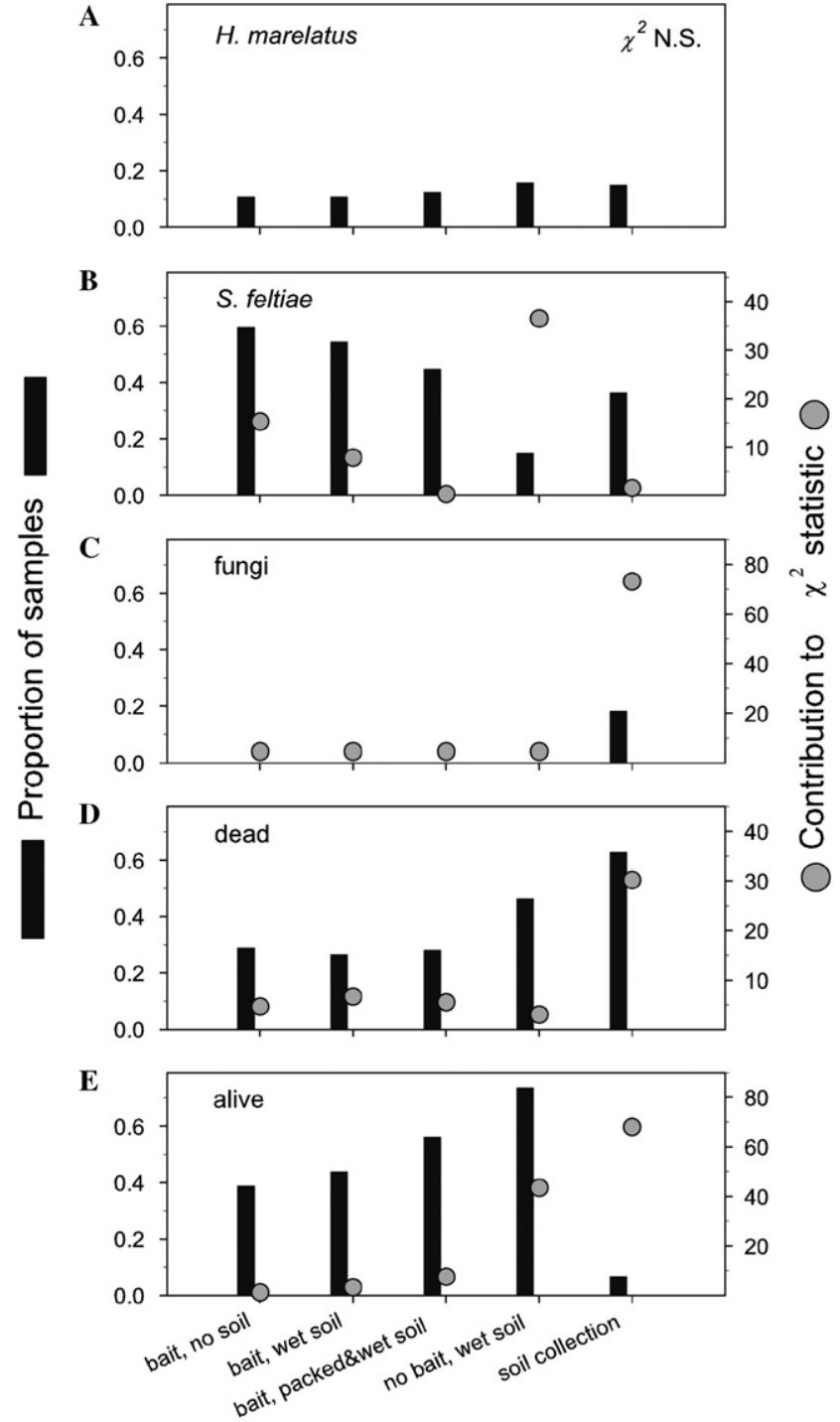

Treatment

Fig. 3. Proportion of sample outcomes observed within baiting treatments from field mesocosms. Outcome categories are: (A) Heterorhabditis marelatus, (B) Steinernema feltiae, (C) entomophagous fungi, (D) Galleria killed by unknown causes, (E) alive Galleria. Outcomes are plotted (black bars) as positive binary responses divided by the total mesocosm replication $(n=121)$. Also plotted is the contribution of each treatment level to the total $\chi^{2}$ statistic (grey bars), when statistically significant. Note that outcomes are plotted on the same relative scale, while $\chi^{2}$ contribution is scaled to the $\chi^{2}$ statistic from each test (Heterorhabditis marelatus, $\chi^{2}=2.296, P=0.681, \mathrm{df}=4$, not shown; all other outcomes $\left.P<0.001\right)$. A high $\chi^{2}$ contribution value shows a disproportionate influence of a treatment level effect on statistical significance of the test.

competition between the two species and reversed the outcome to favor $H$. marelatus.

The high frequency of $H$. marelatus in tubes lacking hosts (tubes baited after removal from soil medium) was surprising. This behavior demonstrated a clear difference from $S$. feltiae, (Fig. 4A). It is possible that $H$. marelatus IJs recruited to the elevated moisture levels in the tubes relative to the surrounding soil medium in both field and

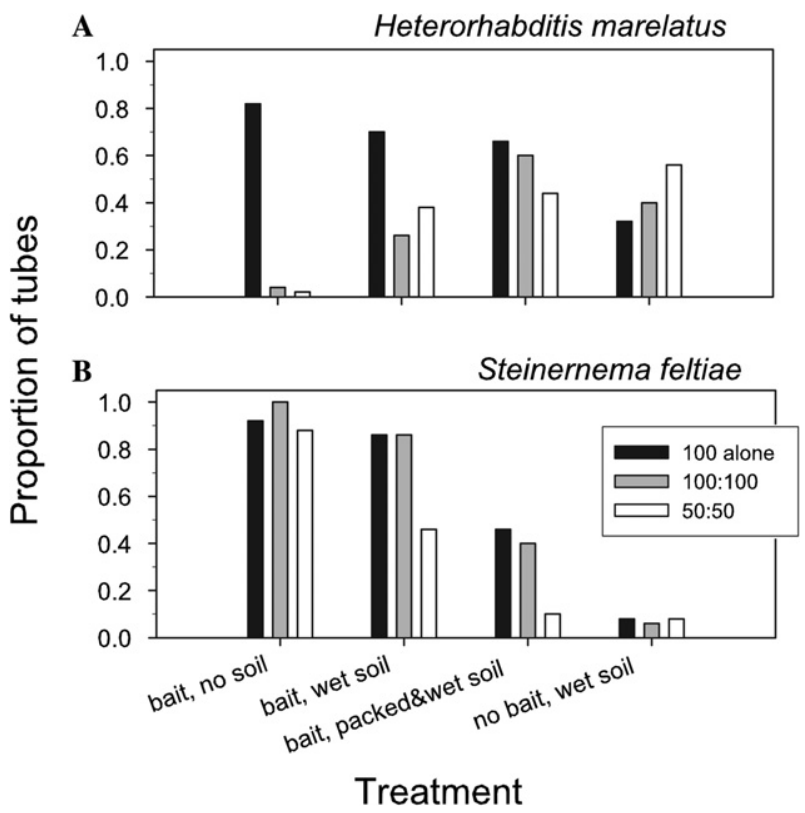

Fig. 4. Proportion of tubes with Galleria mellonella larvae infected by (A) Heterorhabditis marelatus and (B) Steinernema feltiae within lab microcosm treatments in one-species trials (black bars), high-density two-species trials (100:100, grey bars), and low density two-species trials (50:50, white bars). Outcomes are plotted as positive binary responses divided by the total microcosm replication for each species treatment $(n=50)$.

Table 2

Generalized linear model tables for the effects of tube treatments and a heterospecific EPN species on the infection frequency of $H$. marelatus and S. feltiae in laboratory microcosms

\begin{tabular}{lrrllcl}
\hline Source & df & \multicolumn{3}{c}{ Heterorhabditis marelatus } & \multicolumn{2}{c}{ Steinernema feltiae } \\
\cline { 2 - 4 } \cline { 7 - 7 } & & $F$ & $P$ & & $P$ \\
\hline Density sp.2 & 3,490 & 65.382 & $\mathbf{0 . 0 0 0 0}$ & 0.466 & 0.495 \\
Treatment & 2,490 & 10.262 & $\mathbf{0 . 0 0 0 0}$ & & 101.200 & $\mathbf{0 . 0 0 0 0}$ \\
Microcosm & 98,490 & 0.820 & 0.886 & & 1.1621 & 0.156 \\
Tmnt X sp.2 & 6,490 & 13.579 & $\mathbf{0 . 0 0 0 0}$ & 2.893 & $\mathbf{0 . 0 0 9}$ \\
\hline
\end{tabular}

laboratory trials. Numerous studies have demonstrated increased survival and/or persistence as a positive function of soil moisture (e.g., Grant and Villani, 2003; Preisser et al., 2006). We found a trend towards increased movement of $H$. marelatus into unbaited tubes in the presence of $S$. feltiae (Fig. 4A) and a highly significant negative impact of $S$. feltiae on $H$. marelatus infectivity overall. Although heterospecific responses are not always negative (Lewis et al., 2006), these results suggest the hypothesis that $H$. marelatus IJs is repulsed by the cues of ongoing $S$. feltiae infection in tubes with hosts (Grewal et al., 1997; Fairbairn et al., 2000). Because we did not eliminate all organic material from field-collected soils (except by coarse sifting), we cannot determine whether nematodes recruited to carbon dioxide generated by increased microbial activity in wetted soils. Clearly, these hypotheses require further investigation into mechanisms of differing chemotactic responses among species at the behavioral level (Lewis et al., 2006). 
The method of Galleria baiting of soil collections (Bedding and Akhurst, 1975) has allowed huge advances in understanding of the distribution, abundance, and general biology of entomopathogenic nematodes (Kaya and Gaugler, 1993; Gaugler, 2002), and continues to be the gold standard for surveys and isolation of EPNs over large scales. When compared to our in situ baited sampling, however, bait insects in soil collections suffered higher rates of fungal infection and unexplained mortality, and lower incidence of one of two EPN species (Steinernema feltiae, Fig. 3). Previous in situ methods have been proposed (Bedding and Akhurst, 1975; McCoy et al., 2000; Kehres et al., 2001), and we offer an incremental alteration to bait methods that increase the sensitivity, precision, and mechanistic understanding gained from field experiments. Manipulations of sampling tube designs in our study revealed ways to selectively filter one or more EPN species using speciesspecific behavioral traits.

As noted by Bedding and Akhurst (1975), there are disadvantages to using in situ bait sampling methods. Although bait insect loss by predation was eliminated by the tube technique, the fact that sites must be revisited at least once to collect tubes makes in situ sampling less useful for rapid site characterization in remote or large geographic areas. Moreover, in situ sampling relies on active movement of IJs, and may not function as efficiently for surface-dwelling species and strict ambush foragers which do not respond to volatile insect cues (e.g., Steinernema carpocapsae; Lewis et al., 1992, 1993). Tubes also may not perform as well under extreme abiotic conditions (e.g., high or low temperatures, soil moisture) when IJs are less active. Finally, we do not know the size of the sampling arena around traps from which nematodes are drawn. This latter topic is ripe for research, as the 'survey radius' of a trap is likely to vary with soil temperature, porosity, bulk density, physical gradients, nematode species or strain, and the strength of the host signal(s) (Byers and Poinar, 1982; Alatorre-Rosas and Kaya, 1990).

There are numerous distinct advantages to a tube sampling technique for both lab and field experimental studies. As noted above, in situ samplers act as filters to reduce interference and contamination by pathogens, such as entomophagous fungi and nematode-trapping fungi, which are usually present in field collected soils. Entomophagous fungi, such as Beauveria bassiana, rely on host movement but do not forage actively in the soil. Our method thus reduces the incidence of pathogens and fungi that may interfere with EPNs detections. The soil inside tubes may also have buffering capacity in seasonally wet environments: tubes with soil were less likely to be swamped with water, killing the bait insect inside the tube, following a large precipitation event (Gruner, unpublished data). Conversely, preliminary data shows that tube samplers containing moist soil were more sensitive to EPN presence than empty tubes planted in seasonally dry soils (Gruner, unpublished data), perhaps because the moisture seepage from tubes allowed quiescent IJs to move to baits. The tube design allows replicable, precision sampling without soil removal and with minimal soil disturbance while protecting bait insects from scavengers. The design of the tubes as described here also may be useful as a delivery system for IJs from cadavers or titers for applications of nematodes for experimental ecology or pest control functions.

A variety of techniques are needed for the complete ecological toolbox, and our proposed method of EPN in situ sampling adds another flexible option. Nematologists should not be constrained to soil collections as described by Bedding and Akhurst (1975), nor limited to the designs proposed for in situ samplers (McCoy et al., 2000; Kehres et al., 2001). Depending on the purpose of the investigation, modifications of any of the following may be fruitful: the size or porosity of vessels, the characteristics of internal substrate, the identity, biomass, or condition of bait insects, and the duration in the field. For example, in situ field tests of the relative suitability of natural hosts for $H$. marelatus and $S$. feltiae are underway on the Bodega Marine Reserve. Future investigations should estimate the survey radius around sampling points, or the distance from which nematodes are drawn, and determine how this radius varies with soil temperature, porosity, bulk density, physical gradients, nematode species or strain, and the nature or strength of host signals.

\section{Acknowledgments}

We thank Tammie Visintainer for drilling centrifuge tubes, S. Patricia Stock for positively identifying nematode species, Amanda Hodson, Glen Stevens, Evan Preisser, and two anonymous reviewers for valuable comments, and the National Science Foundation for financial support (DEB0620475). This is contribution number 2352 of the Bodega Marine Lab, University of California at Davis.

\section{References}

Alatorre-Rosas, R., Kaya, H.K., 1990. Interspecific competition between entomopathogenic nematodes in the genera Heterorhabditis and Steinernema for an insect host in sand. J. Invert. Pathol. 55 (2), 179-188.

Anderson, R.M., May, R.M., 1981. The population dynamics of microparasites and their invertebrate hosts. Phil. Trans. R. Soc. B 291, 451-524.

Barbercheck, M.E., Kaya, H.K., 1991. Effect of host condition and soil texture on host finding by the entomogenous nematodes Heterorhabditis bacteriophora (Rhabditida: Heterorhabiditidae) and Steinernema carpocapsae (Rhabditida: Steinernematidae). Environ. Entomol. 20 (2), 582-589.

Barbour, M.G., Craig, R.B., Drysdale, F.R., Ghiselin, M.T., 1973. Coastal Ecology: Bodega Head. University of California Press, Berkeley, CA. p. 338.

Bedding, R.A., Akhurst, R.J., 1975. A simple technique for the detection of insect parasitic rhabditid nematodes in soil. Nematologica 21, 109-116.

Blackshaw, R.P., Senthamizhselvan, M., 1991. The effect of sand particlesize on Steinernema feltiae sensu Filipjev (1934) (=N. bibionis Bovien) activity against Galleria mellonella larvae. Annals of Applied Biology 118 (3), 637-643.

Brown, I.M., Gaugler, R., 1997. Temperature and humidity influence emergence and survival of entomopathogenic nematodes. Nematologica 43 , 363-375. 
Byers, J.A., Poinar Jr., G.O., 1982. Location of insect hosts by the nematode, Neoaplectana carpocapsae, in response to temperature. Behaviour 79 (1), 1-10.

Fairbairn, J.P., Fenton, A., Norman, R.A., Hudson, A.J., 2000. Re-assessing the infection strategies of the entomopathogenic nematode Steinernema feltiae (Rhabditidae; Steinernematidae). Parasitology 121 (2), 211-216.

Fan, X., Hominick, W.M., 1991. Efficiency of the Galleria (wax moth) baiting technique for recovering infective stages of entomopathogenic rhabditids (Steinernematidae and Heterorhabditidae) from sand and soil. Revue de Nematologie 14 (3), 381-387.

Gaugler, R. (Ed.), 2002. Entomopathogenic Nematology. CABI Publishing., London.

Gaugler, R., LeBeck, L., Nakagaki, B., Boush, G.M., 1980. Orientation of the entomogenous nematode Neoaplectana carpocapsae to carbon dioxide. Environ. Entomol. 9, 649-652.

Georgis, R., Poinar Jr., G.O., 1983a. Effect of soil texture on the distribution and infectivity of Neoaplectana carpocapsae (Nematoda: Steinernematidae). J. Nemat. 15 (2), 308-311.

Georgis, R., Poinar Jr., G.O., 1983b. Effect of soil texture on the distribution and infectivity of Neoaplectana glaseri (Nematoda: Steinernematidae). J. Nemat. 15 (2), 329-332.

Gouge, D.H., Lee, L.L., Henneberry, T.J., 1999. Effect of temperature and Lepidopteran host species on entomopathogenic nematode (Nematoda: Steinernematidae, Heterorhabditidae) infection. Environ. Entomol. 28 (5), 876-883.

Grant, J.A., Villani, M.G., 2003. Soil moisture effects on entomopathogenic nematodes. Environ. Entomol. 32 (1), 80-87.

Grewal, P.S., Lewis, E.E., Gaugler, R., 1997. Response of infective stage parasites (Nematoda: Steinernematidae) to volatile cues from infected hosts. J. Chem. Ecol. 23 (2), 503-515.

Hass, B., Griffin, C.T., Downes, M.J., 1999. Persistence of Heterorhabditis infective juveniles in soil: comparison of extraction and infectivity measurements. J. Nemat. 31 (4), 508-516.

Hui, E., Webster, J.M., 2000. Influence of insect larvae and seedling roots on the host-finding ability of Steinernema feltiae (Nematoda: Steinernematidae). J. Invert. Pathol. 75, 152-162.

Hunt, H.W., Wall, D.H., DeCrappeo, N.M., Brenner, J.S., 2001. A model for nematode locomotion in soil. Nematology 3 (7), 705-716.

Jaffee, B.A., Strong, D.R., 2005. Strong bottom-up and weak top-down effects in soil: nematode-parasitized insects and nematode-trapping fungi. Soil Biol. Biochem. 37, 1011-1021.

Kaya, H.K., Gaugler, R., 1993. Entomopathogenic nematodes. Ann. Rev. Entomol. 38, 181-206.

Kehres, J., Denon, D., Mauléon, H., 2001. A simple technique to estimate, in situ, population densities of an entomopathogenic nematode (Haeterorhabditis indica) in sandy soils. Nematology 3 (3), 285-287.

Koppenhöfer, A.M., Fuzy, E.M., 2006. Effect of soil type on infectivity and persistence of the entomopathogenic nematodes Steinernema scarabaei, Steinernema glaseri, Heterorhabditis zealandica, and Heterorhabditis bacteriophora. J. Invert. Pathol. 92 (1), 11-22.

Koppenhöfer, A.M., Campbell, J.F., Kaya, H.K., Gaugler, R., 1998. Estimation of entomopathogenic nematode population density in soil by correlation between bait insect mortality and nematode penetration. Fund. Appl. Nemat. 21 (1), 95-102.

Kung, S.-P., Gaugler, R., Kaya, H.K., 1990. Soil type and entomopathogenic nematode persistence. J. Invert. Pathol. 55, 401-406.

Kung, S.-P., Gaugler, R., Kaya, H.K., 1991. Effects of soil temperature, moisture, and relative humidity on entomopathogenic nematode persistence. J. Invert. Pathol. 57, 242-249.

Lewis, E.E., Gaugler, R., Harrison, R., 1992. Entomopathogenic nematode host finding: response to host contact cues by cruise and ambush foragers. Parasitology 105, 309-315.
Lewis, E.E., Gaugler, R., Harrison, R., 1993. Response of cruiser and ambusher entomopathogenic nematodes (Steinernematidae) to host volatile cues. Can. J. Zool. 71, 765-769.

Lewis, E.E., Campbell, J., Griffin, C., Kaya, H., Peters, A., 2006. Behavioral ecology of entomopathogenic nematodes. Biol. Control 38 (1), 66-79.

McCoy, C.W., Shapiro, D.I., Duncan, L.W., Nguyen, K., 2000. Entomopathogenic nematodes and other natural enemies as mortality factors for larvae of Diaprepes abbreviatus (Coleoptera: Curculionidae). Biol. Control 19, 182-190.

Miller, V.C. (Eds), 1972. Soil Survey of Sonoma County, California. Forest Soil and Soil Conservation Service, United States Department of Agriculture.

Neher, D.A., Weicht, T.R., Moorhead, D.L., Sinsabaugh, R.L., 2004. Elevated $\mathrm{CO}_{2}$ alters functional attributes of nematode communities in forest soils. Funct. Ecol. 18 (4), 584-591.

Nielsen, O., Skovgaard, I.M., Philipsen, H., 2004. Estimating the incidence of entomopathogenic nematodes in soil by the use of bait insects. Nematology 6 (6), 891-900.

O'Halloran, D.M., Burnell, A.M., 2003. An investigation of chemotaxis in the insect parasitic nematode Heterorhabditis bacteriophora. Parasitology $127,375-385$.

Poinar Jr., G.O., 1990. Taxonomy and biology of Steinernematidae and Heterorhabditidae. In: Gaugler, R., Kaya, H.K. (Eds.), Entomopathogenic Nematodes in Biological Control. CRC Press, Boca Raton, FL, pp. 23-61.

Portillo-Aguilar, C., Villani, M.G., Tauber, M.J., Tauber, C.A., Nyrop, J.P., 1999. Entomopathogenic nematode (Rhabditida: Heterorhabditidae and Steinernematidae) response to soil texture and bulk density. Environ. Entomol. 28 (6), 1021-1035.

Preisser, E.L., Dugaw, C.J., Dennis, B., Strong, D.R., 2006. Plant facilitation of a belowground predator. Ecology 87 (5), 1116-1123.

Rasmann, S., Köllner, T.G., Degenhardt, J., Hiltpold, I., Toepfer, S., Kuhlmann, U., Gershenzon, J., Turlings, T.C.J., 2005. Recruitment of entomopathogenic nematodes by insect-damaged maize roots. Nature 434, 732-737.

Schmidt, J., All, J.N., 1978. Chemical attraction of Neoaplectana carpocapsae (Nematoda: Steinernematidae) to insect larvae. Environ. Entomol. 7 (4), 605-607.

Schmidt, J., All, J.N., 1979. Attraction of Neoaplectana carpocapsae (Nematoda: Steinernematidae) to common excretory products of insects. Environ. Entomol. 8 (1), 55-61.

Stock, S.P., 1997. Heterorhabditis hepialus Stock, Strong \& Gardner, 1996: a junior synonym of $H$. marelatus Liu \& Berry, 1996 (Rhabditida: Heterorhabditidae) with a redescription of the species. Nematologica 43, $455-463$.

Stock, S.P., Strong, D., Gardner, S.L., 1996. Identification of Heterorhabditis (Nematoda: Heterorhabditidae) from California with a new species isolated from the larvae of the ghost moth Hepialus californicus (Lepidoptera: Hepialidae) from the Bodega Bay Natural Reserve. Fund. Appl. Nemat. 19 (6), 585-592.

Stock, S.P., Pryor, B.M., Kaya, H.K., 1999. Distribution of entomopathogenic nematodes (Steinernematidae and Heterorhabditidae) in natural habitats in California, USA. Biodiv. Conserv. 8, 535-549.

Strong, D.R., Kaya, H.K., Whipple, A.L., Child, A.L., Kraig, S., Bondonno, M., Dyer, K., Maron, J.L., 1996. Entomopathogenic nematodes: natural enemies of root-feeding caterpillars on bush lupine. Oecologia 108, $167-173$.

Woodring, J.L., Kaya, H.K., 1998. Steinernematid and Heterorhabditid Nematodes: a Handbook of Techniques. Southern Cooperative Series Bulletin, 331. Arkansas Agricultural Experiment Station, Fayetteville, Arkansas, 30 pp. 